\title{
UBM/slit-lamp-photo imaging of pseudoexfoliation deposits in the iridocorneal angle: imaging clues to the genesis of ocular hypertension
}

\author{
V. Tao Tran
}

Received: 23 January 2008/Accepted: 1 July 2008/Published online: 24 July 2008

(C) The Author(s) 2008

\begin{abstract}
This photo essay is aimed at showing slitlamp photographic views and its ultrasound biomicroscopy (UBM) corollaries of angle deposits in pseudoexfoliation syndrome cases and contributes visual arguments to the hypotheses and explanations of the genesis of ocular hypertension.
\end{abstract}

Keywords Ultrasound biomicroscopy ·

Pseudoexfoliation · Iridocorneal angle

\section{Case report}

Pseudoexfoliation (PEX) was first described in 1917 by Lindberg [1]. One of the consequences of PEX is ocular hypertension and glaucoma [2], which becomes increasingly frequent with age [3]. Blockage of the trabecular meshwork by the PEX material decreases aqueous humour outflow and increases consequently ocular tension [4]. Jacobi introduced a technique called trabecular aspiration to treat PEX glaucoma [5].

For several years, we have followed patients and correlated slit-lamp photography with ultrasound biomicroscopy (UBM) images of PEX deposits in the iridocorneal angle (ICA). The many images obtained and the sequential analysis of PEX deposits

V. Tao Tran $(\bowtie)$

Centre for Ophthalmic Specialized Care (COS),

9, Ave Beaumont, Lausanne, Switzerland

e-mail: trantao@citycable.ch in the angle before and after cataract surgery inspired this photo essay and could represent evidence on the events that can lead to ocular hypertension.

We show a case of an 81-year-old woman followed in our centre for PEX syndrome of the left eye with abundant PEX material on the pupillary border (Fig. 1a) and in the ICA (Fig. 2a) as well as a cataract. Ocular tension was elevated to $24 \mathrm{mmHg}$ despite antihypertensive drops. Exploration of the ICA, especially its lower $180^{\circ}$, was done by UBM (Fig. 3a). Standard cataract surgery with the intraocular lens implantation in the capsular bag was carried out without complications. Special care was given to a thorough washout of PEX material on $360^{\circ}$ in the ICA, especially its bottom part. Postoperatively ocular pressure decreased to $11 \mathrm{mmHg}$ on the first postoperative day and remained within the norm during a 1-year of follow-up without any treatment.

Two, 6, and 9 months after cataract surgery, we carried out slit-lamp photography of the same structures showing the absence of PEX material on the pupillary border, surface of the iris (Fig. 1b), and in the ICA (Fig. 2a, b). UBM after cataract surgery also showed that PEX material disappeared in the ICA (Fig. 3b).

\section{Comment}

In this case, abundant PEX material on the pupillary margin overflowed onto the iris surface and 
Fig. 1 a Pseudoexfoliation (PEX) material overflows on the pupillary border and on the iris surface.

b Absence of PEX material on the pupillary border and on the iris surface after 2 months of cataract surgery
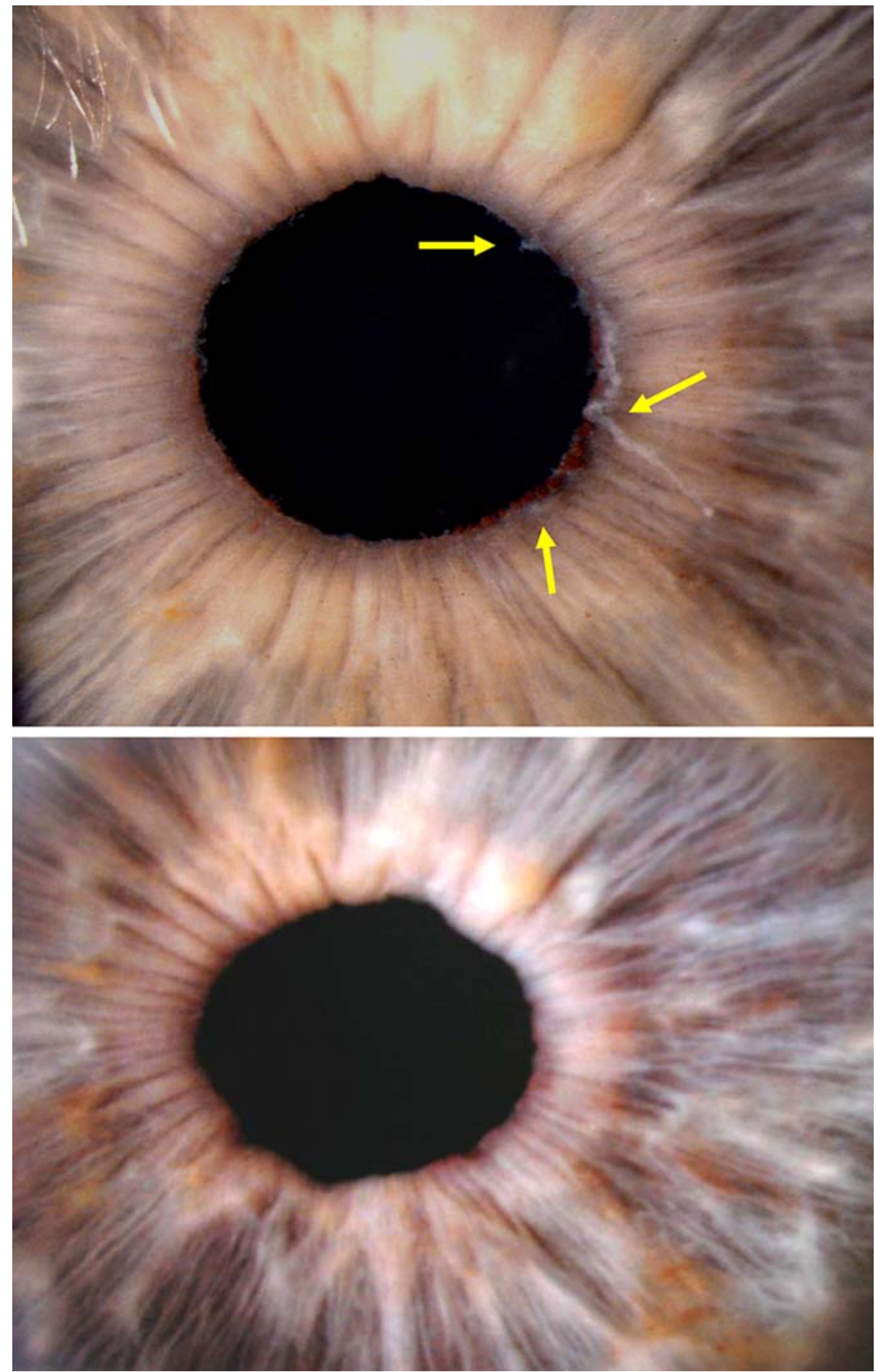

sedimented to the bottom of the ICA. UBM examination confirmed the presence of PEX material in the bottom of the ICA but not in its upper part. Localization of PEX material in many places in the anterior chamber indicates the large amount of material that can be generated and this material finally gets into the lower part of the ICA.

It is probably this mechanical accumulation of PEX material within the ICA, very clearly demonstrated in this case by slit-lamp photography and 
Fig. 2 a Pseudoexfoliation (PEX) deposits in the iridocorneal angle (ICA). Before cataract surgery, the ICA shows the presence of PEX covering most of the vessels hidden behind the PEX material in the ICA. b Two months after cataract surgery, PEX material disappeared and the vessels appeared in the ICA
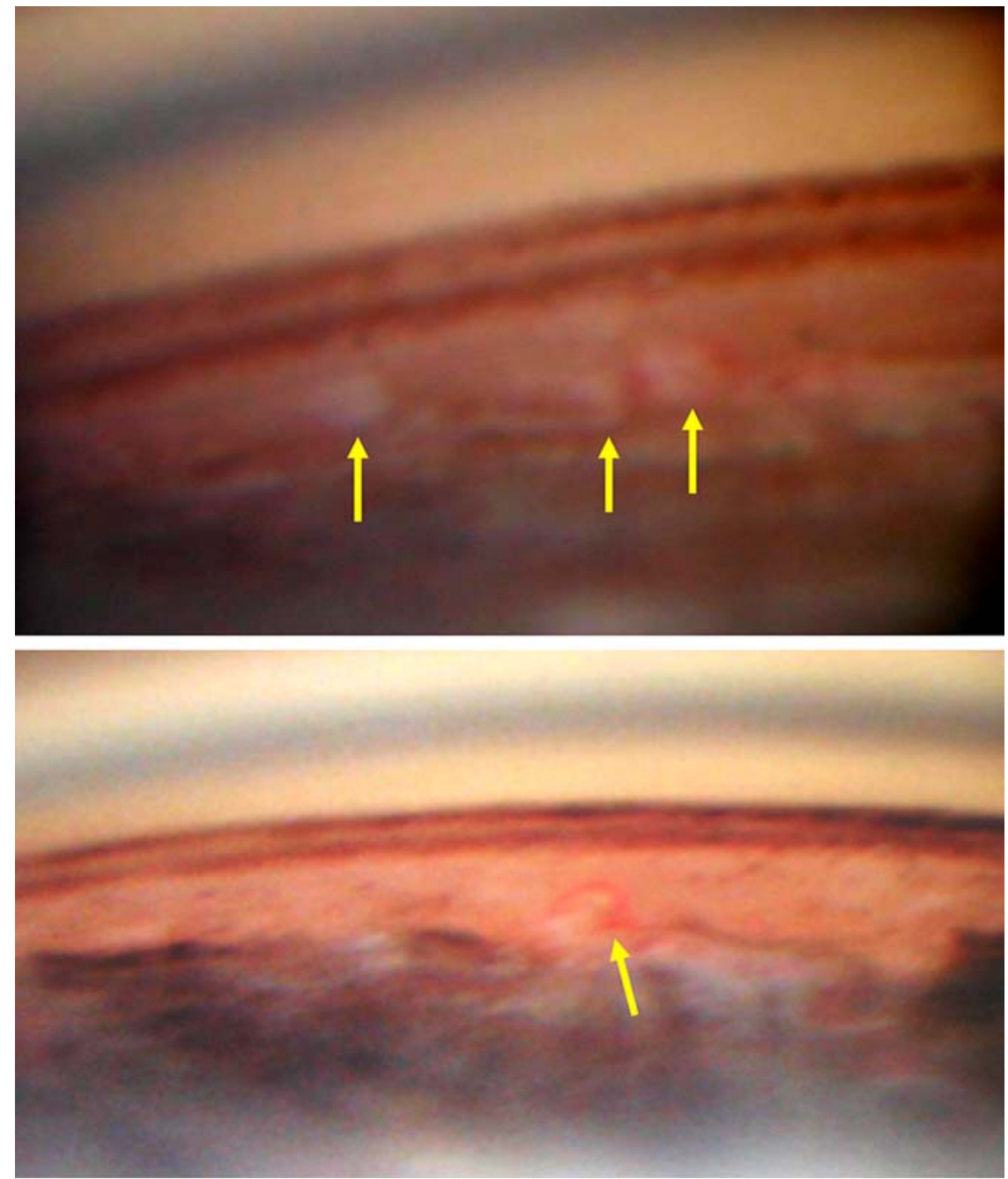

UBM, that produces the rise of intraocular pressure. This mechanical thesis seems to be in most cases the sole reason for the increase of pressure, as mechan- ical washout of the PEX material during cataract surgery seems to solve the problem. 
Fig. 3 a Before cataract surgery, pseudoexfoliation (PEX) material in the iridocorneal angle (ICA) shown by ultrasound biomicroscopy (UBM). b Three months after cataract surgery, PEX material disappeared in the ICA
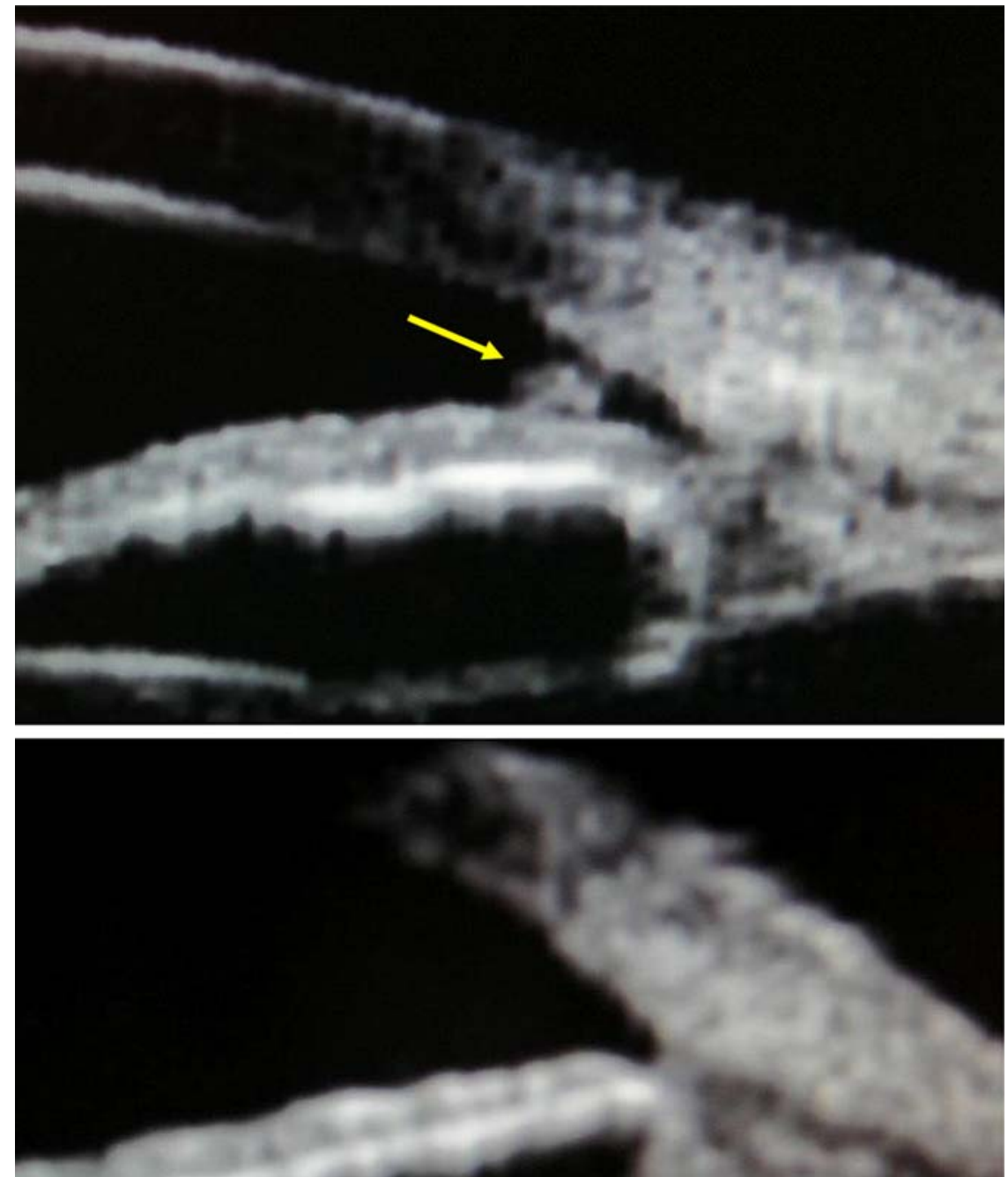

Open Access This article is distributed under the terms of the Creative Commons Attribution Noncommercial License which permits any noncommercial use, distribution, and reproduction in any medium, provided the original author(s) and source are credited.

\section{References:}

1. Lindberg JG (1917) Clinical studies of depigmentation of the pupillary margin and transillumination of the iris in cases of senile cataract and also in normal eyes in the aged (Thesis). Helsinki University, Helsinki, Finland; Kliniska undersokningar over depigmenteringen av pupillarranden och genomlysbarheten av iris vid fall av aldersstarr samt $\mathrm{i}$ normala ogon hos gamla personer

2. Ritch R (1994) Exfoliation syndrome: The most common identifiable cause of open-angle glaucoma. Trans Am Ophthalmol Soc 92:845-944

3. Mitchell P, Wang JJ, Hourihan F (1999) The relationship between glaucoma and pseudoexfoliation: the Blue Mountains eye study. Arch Ophthalmol 117(10):1319-1324

4. Haydon PR (1986) Pseudoexfoliation syndrome as a cause of chronic glaucoma. Klin Monatsbl Augenheilkd 189:293-301

5. Jacobi PC, Dietlein TS, Krieglstein GK (1998) Bimanual trabecular aspiration in pseudoexfoliation glaucoma: an alternative in nonfiltering glaucoma surgery. Ophthalmology 105(5):886-894 